\title{
Autophagy regulates adipose mass and differentiation in mice
}

\author{
Rajat Singh, ${ }^{1,2}$ Youqing Xiang,, ${ }^{1,2}$ Yongjun Wang,1,2 Kiran Baikati,,1,2 Ana Maria Cuervo,1,2,3,4,5 \\ Yen K. Luu,1,4 Yan Tang,1,4 Jeffrey E. Pessin,1,4 Gary J. Schwartz,1,4,6 and Mark J. Czaja1,2,4
}

1Department of Medicine, ${ }^{2}$ Marion Bessin Liver Research Center, ${ }^{3}$ Department of Developmental and Molecular Biology, ${ }^{4}$ Diabetes Research and Training Center, ${ }^{5}$ Institute for Aging Studies, and ${ }^{6}$ Department of Neuroscience, Albert Einstein College of Medicine, New York, New York, USA.

The relative balance between the quantity of white and brown adipose tissue can profoundly affect lipid storage and whole-body energy homeostasis. However, the mechanisms regulating the formation, expansion, and interconversion of these 2 distinct types of fat remain unknown. Recently, the lysosomal degradative pathway of macroautophagy has been identified as a regulator of cellular differentiation, suggesting that autophagy may modulate this process in adipocytes. The function of autophagy in adipose differentiation was therefore examined in the current study by genetic inhibition of the critical macroautophagy gene autophagy-related 7 (Atg7). Knockdown of Atg7 in 3T3-L1 preadipocytes inhibited lipid accumulation and decreased protein levels of adipocyte differentiation factors. Knockdown of Atg5 or pharmacological inhibition of autophagy or lysosome function also had similar effects. An adipocyte-specific mouse knockout of Atg7 generated lean mice with decreased white adipose mass and enhanced insulin sensitivity. White adipose tissue in knockout mice had increased features of brown adipocytes, which, along with an increase in normal brown adipose tissue, led to an elevated rate of fatty acid, $\beta$-oxidation, and a lean body mass. Autophagy therefore functions to regulate body lipid accumulation by controlling adipocyte differentiation and determining the balance between white and brown fat.

\section{Introduction}

Obesity is characterized by an expansion of adipose tissue mass resulting from increased adipocyte number and/or size. Lipids in the form of triglycerides (TG) accumulate in various anatomical locations that differ in several regards including whether they are composed primarily of white or brown adipocytes. These 2 distinct types of adipocytes differ in their lipid content and metabolic functions. White adipose tissue (WAT) serves the primary function of lipid storage in the fed state and with fasting releases fatty acids from the breakdown of TG into the circulation for muscle energy production. In contrast, brown adipose tissue (BAT) has more limited TG storage and does not secrete fatty acids but instead uses them for autonomous energy expenditure and heat generation (1). Although the amount of BAT in adult humans has been previously considered to be minimal, recent findings of significant concentrations of brown adipocytes in adult humans $(2-5)$ have raised the possibility that the balance between WAT and BAT mass may be one factor that regulates the development of obesity and its severity (6). Manipulating the process of adipocyte differentiation in order to promote more BAT in place of WAT may therefore be a novel approach to the treatment of obesity and its associated problems (7).

Factors determining the differential development of WAT versus BAT remain poorly defined. In mammals, WAT and BAT generally develop before birth, although in rodents, WAT develops postnatally (8). Recent studies suggest that these fat cell populations are not static and may in fact continue to undergo significant cell turnover (9). A long-standing belief has been that white and brown adipocytes develop from a common precursor cell. However, recent evidence suggests that they are in fact developmentally distinct, and brown adipocytes may share a common precursor

Conflict of interest: The authors have declared that no conflict of interest exists. Citation for this article: J. Clin. Invest. 119:3329-3339 (2009). doi:10.1172/JCI39228. with skeletal muscle cells rather than white adipocytes $(10,11)$. The loss of BAT after birth, along with the ability to increase BAT under certain physiological conditions such as low ambient temperature (8), has suggested that adipocytes may transdifferentiate from one type into the other. However, a mechanism for this process has not been delineated.

Macroautophagy is a nonselective lysosomal pathway for the degradation of cytosolic organelles and proteins. In this highly regulated process, whole organelles and portions of cytosol are sequestered inside double-membrane vesicles termed autophagosomes that translocate to lysosomes for fusion and content degradation. In addition to its established roles in regulating nutrient supply and cell death, autophagy also functions in cellular development and differentiation. Studies of mice with global knockouts of critical autophagy genes have revealed that developmental defects occur in the absence of autophagy, particularly in the nervous system (12). These findings have given rise to the concept that autophagy may regulate development by performing critical cellular remodeling functions required for differentiation (12).

To determine the function of autophagy in adipose differentiation, the effects of an in vitro knockdown and a selective in vivo knockout of the critical macroautophagy gene autophagy-related 7 (Atg7) in mouse adipocytes were examined. Loss of Atg7 in 3T3-L1 preadipocytes resulted in an inhibition of autophagic function and decreased TG accumulation and expression of markers of adipocyte differentiation. Adipocyte-selective knockout mice displayed a lean phenotype due to a marked reduction in WAT mass and a conversion from a white adipocyte to brown adipocyte phenotype in WAT along with an increase in BAT mass. This increase in brown adipocytes resulted in improved insulin sensitivity and increased fatty acid $\beta$-oxidation. These findings demonstrate a critical function for autophagy in adipocyte development and differentiation that determines the balance between WAT and BAT. 
A

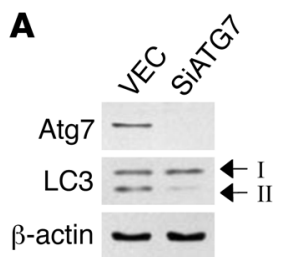

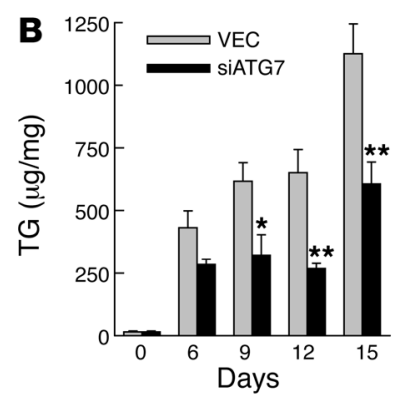

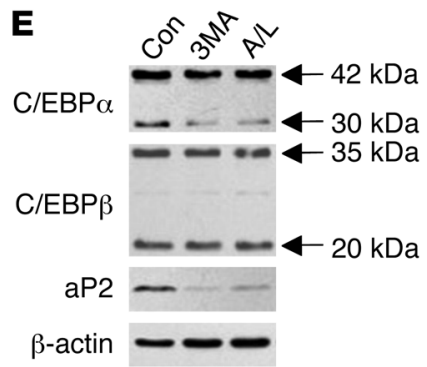

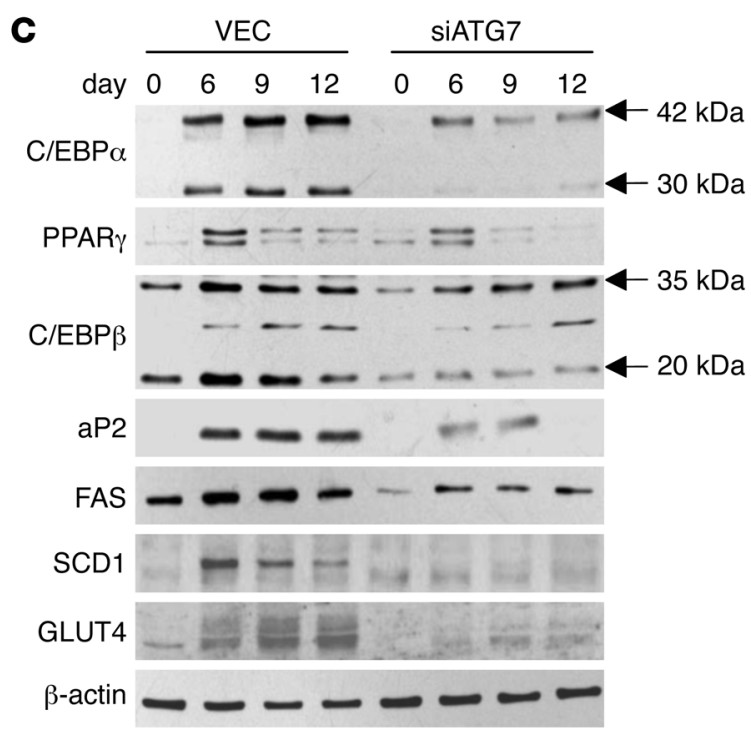

\section{Figure 1}

An inhibition of autophagy blocks 3T3-L1 cell TG accumulation and differentiation. (A) Total protein was isolated from VEC and siATG7 cells and immunoblotted with antibodies for Atg7, LC3, and $\beta$-actin. The LC3-I and LC3-II forms are indicated. (B) TG levels in VEC and siATG7 cells at the days indicated after initiation of adipocyte differentiation. Results are represented as mean $+\operatorname{SEM}(n=4-8)$. ${ }^{\star} P<0.02$; ${ }^{\star \star} P<0.006$ compared with VEC cells. (C) Immunoblots of proteins isolated from VEC and siATG7 cells on the indicated days of differentiation and probed with the antibodies shown. (D) TG levels on day 6 of differentiation in control, untreated wild-type cells (Con) and cells treated with 3-methyladenine $(3 \mathrm{MA})$ or ammonium chloride/leupeptin (A/L). Results are shown as mean $+\mathrm{SEM}(n=6-9)$. ${ }^{\star} P<0.0006$ compared with untreated cells. (E) Immunoblots of day 6 control and 3-methyladenine- and ammonium chloride/leupeptin-treated cells.

\section{Results}

Inhibition of autophagy blocks 3T3-L1 cell lipid accumulation and differentiation. To determine whether autophagy functions in the process of adipocyte differentiation, levels of TG and protein markers of adipocyte differentiation were examined in 3T3-L1 cells with an inhibition of autophagy. 3T3-L1 cells stably infected with empty lentiviral vector (VEC cells) or with vector expressing an shRNA to the essential autophagy gene Atg7 (siATG7 cells) were induced to undergo adipocyte differentiation. Levels of Atg7 were markedly decreased on immunoblots in siATG7 cells in parallel with reduced levels of the conjugated form of microtubule-associated light chain 3 (LC3), LC3-II, compatible with a decrease in autophagic function (Figure 1A). A block in macroautophagy was confirmed by measure of autophagic flow as determined by the change in levels of LC3-II between untreated cells and cells treated with the lysosomal inhibitors ammonium chloride and leupeptin (13). LC3-II levels increased markedly in inhibitor-treated VEC cells but not siATG7 cells when compared with untreated cells, indicating that the Atg7 knockdown was effective in blocking macroautophagy (Supplemental Figure 1A; supplemental material available online with this article; doi:10.1172/JCI39228DS1).

Cellular TG levels were examined over time as the VEC and siATG7 cells differentiated into adipocytes. Undifferentiated VEC cells (day 0) had minimal TG stores that increased markedly with differentiation (day 6) and further increased with maintenance in culture (days 6-15; Figure 1B). siATG7 cells started similarly with low baseline TG levels that increased with differentiation; however, cellular TG content was significantly decreased in these cells compared with VEC cells (Figure 1B). The lack of TG accu- mulation was not secondary to cell death from the Atg7 knockdown, as steady-state levels of apoptosis and necrosis measured by fluorescence microscopic studies of acridine orange- and ethidium bromide-costained cells were equivalent in the 2 cell types after the induction of differentiation (Supplemental Figure 2A). To insure that these findings were reflective of an effect on macroautophagy, similar studies were performed in 3T3-L1 cells with a knockdown of a second autophagy gene, Atg5 (Supplemental Figure 3A). An Atg5 knockdown from 2 different shRNAs also significantly decreased TG levels in cells that were induced to differentiate (Supplemental Figure 3B).

The finding that an inhibition of autophagy limited the TG accumulation characteristic of adipocytes suggested that 3T3-L1 cell adipogenic differentiation had been blocked. Consistent with this possibility was the fact that protein levels of mediators of 3T3-L1 cell adipocyte differentiation (6) were decreased in concert with the reduced lipid accumulation in siATG7 cells. Marked increases in CCAAT/enhancer binding protein $\alpha$ $(\mathrm{C} / \mathrm{EBP} \alpha)$ and PPAR $\gamma$ were detected in VEC cells on day 6 by immunoblotting (Figure 1C). Induction of C/EBP $\alpha$ and PPAR $\gamma$ also occurred in siATG7 cells, but levels were markedly less than in VEC cells (Figure 1C). Levels of C/EBP $\beta$ were also decreased in siATG7 cells relative to VEC cells (Figure 1C). In addition, markers of adipocyte differentiation including aP2 (14), fatty acid synthase (FAS), stearoyl-coenzyme A desaturase 1 (SCD1), and GLUT4 either were not induced or were induced to a much lesser extent in siATG7 cells. Similar effects on mediators and markers of adipocyte differentiation occurred in Atg5-knockdown cells (Supplemental Figure 3C). 
A

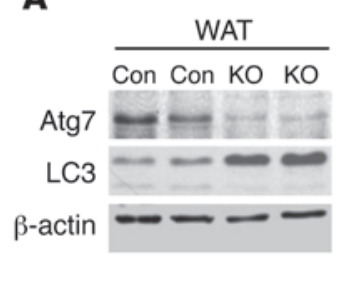

C

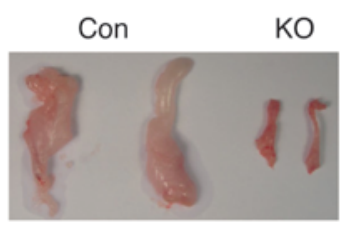

$\mathbf{F}$

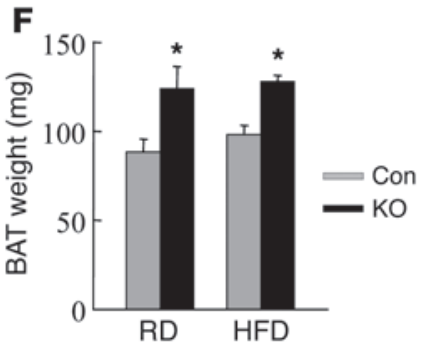

BAT

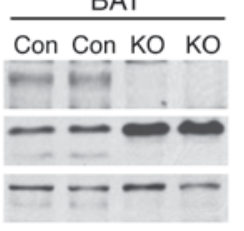

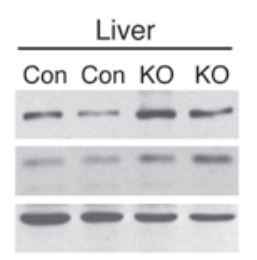

B

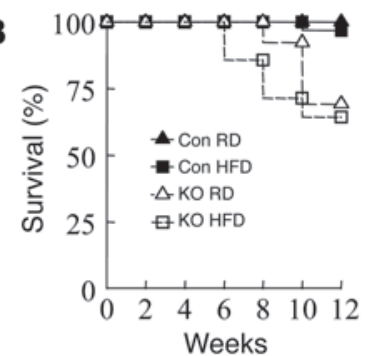

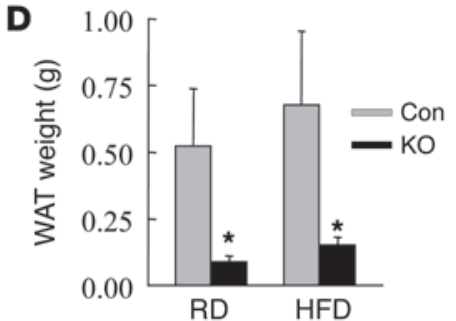

G

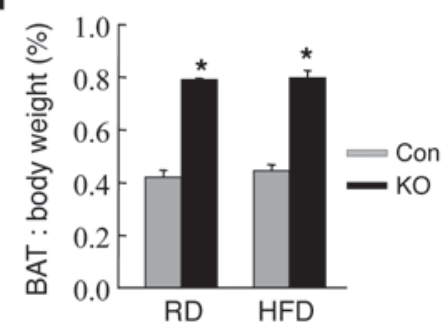

E

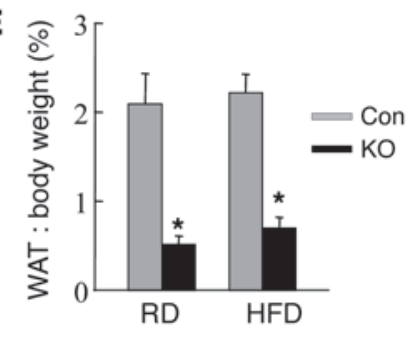

H

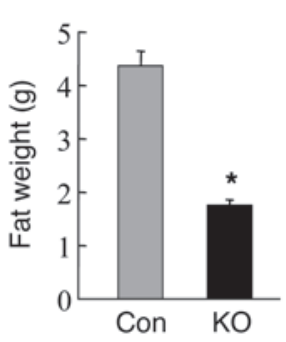

I

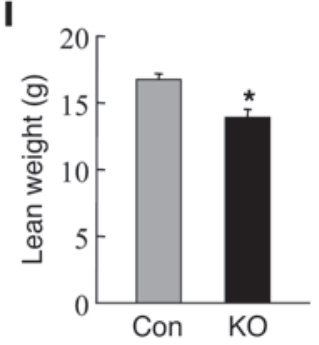

Figure 2

ATG7F/F-aP2-Cre mice have decreased WAT and increased BAT mass. (A) Immunoblots of proteins isolated from the indicated tissues from control (Con) ATG7F/F and knockout ATG7F/F-aP2-Cre mice. The LC3-I and LC3-II forms are indicated. (B) Survival curves for RD- and HFDfed mice $(n=13-28)$. (C) Representative gonadal fat pads from the 2 types of mice. (D) Gonadal WAT weights in RD- and HFD-fed mice $(n=5-14)$. ${ }^{\star} P<0.001$. (E) WAT weight as a percentage of total body weight $(n=4-24)$. ${ }^{\star} P<0.005$. (F) Interscapular BAT weights $(n=3-17)$. ${ }^{\star} P<0.05$. (G) BAT weight as a percentage of total body weight $(n=3-17)$. ${ }^{*}<0.00002$. (H) Total fat mass as determined by magnetic resonance spectroscopy $(n=8-20)$. ${ }^{*} P<0.00001$. (I) Lean body mass in the same animals $(n=8-20)$. ${ }^{\star} P<0.002$. Results are mean + SEM. $P$ values are as compared with control animals.

The function of autophagy in 3T3-L1 cell differentiation was further confirmed by studies with pharmacological inhibitors. 3T3-L1 cells received a single treatment of 3-methyladenine, an inhibitor of autophagy (15), at the time of initiation (day 0). Treatment with 3-methyladenine blocked macroautophagy, as determined by measure of autophagic flow. Decreased autophagic flow in 3-methyladenine-treated cells was demonstrated by a decreased ratio of LC3-II in cells in the presence of lysosomal inhibitors to cells in the absence of inhibitors as compared with the ratio in cells without 3-methyladenine treatment (Supplemental Figure 1B). Inhibition of autophagic function at the time of initiation of adipocyte differentiation significantly decreased subsequent cellular TG accumulation (day 6; Figure 1D). Treatment with 3-methyladenine had no effect on cell viability (Supplemental Figure 2B). Treatment with 3-methyladenine also altered levels of adipocyte differentiation factors, although the effects were not as significant as with the genetic knockdown. Decreased levels of the $30-\mathrm{kDa}$ form of C/EBP $\alpha$ along with a marked reduction in aP2 occurred in 3-methyladenine-treated cells (Figure 1E). Similar decreases in TG content and $\mathrm{aP} 2$ and $\mathrm{C} / \mathrm{EBP} \alpha$ levels were seen with nonspecific lysosomal inhibition with ammonium chloride and leupeptin (Figure $1, \mathrm{D}$ and $\mathrm{E})$. Thus, both genetic and pharmacological inhibitor studies demonstrated that autophagy had a critical function in the process of adipocyte differentiation and lipid accumulation in 3T3-L1 preadipocytes.

Adipocyte-specific Atg7-knockout mice are lean and have reduced WAT mass. To determine whether findings in 3T3-L1 cells reflected the in vivo function of macroautophagy in adipocytes, ATG7 $7^{\mathrm{F}} \mathrm{F}-\mathrm{aP} 2-\mathrm{Cre}$ mice containing an adipocyte-selective knockout of Atg7 were generated. The tissue specificity of the Atg 7 knockout was confirmed by immunoblotting that demonstrated the absence of Atg7 and LC3-II, along with a compensatory increase in LC3-I, in gonadal WAT and interscapular BAT but not other organs (Figure 2A). The number of ATG7 ${ }^{\mathrm{F} / \mathrm{F}}$-aP2-Cre births was slightly decreased from the expected Mendelian ratio (cross of ATG7F/F $\times$ ATG7F/WT-aP2-Cre yielded $18 \% \mathrm{ATG}^{\mathrm{F} / \mathrm{F}}$-aP2-Cre mice; $n=88$ ). Furthermore, after 6 weeks of life, ATG7 $7^{\mathrm{F} / \mathrm{F}}$-aP2-Cre mice on regular diet (RD) or highfat diet (HFD) had higher spontaneous death rates than littermate control ATG $7^{\mathrm{F} / \mathrm{F}}$ mice (Figure $2 \mathrm{~B}$ ). Loss of autophagic function in adipose tissue therefore led to an increase in mortality.

Body-weight analysis revealed that $\mathrm{ATG} 7^{\mathrm{F} / \mathrm{F}}$-aP2-Cre mice weighed on average $27 \%$ less than control ATG7 $7^{\mathrm{F} / \mathrm{F}}$ mice on RD and $37 \%-45 \%$ less when fed HFD from the ages of 8 to 12 weeks (Supplemental Figure 4, A and B). ATG7 $7^{\mathrm{F} / \mathrm{F}}$-aP2-Cre mice gained weight on either diet but maintained their reduction in weight 

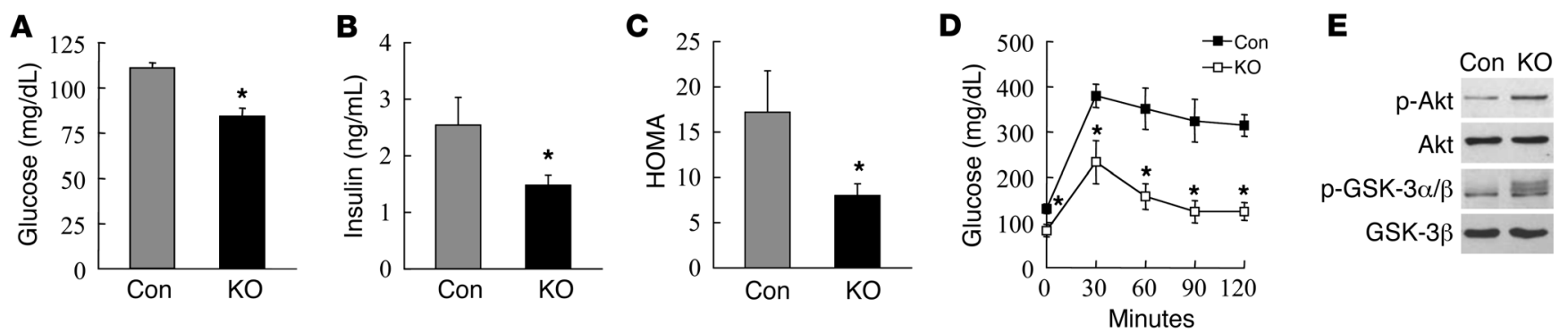

Figure 3

HFD-fed ATG7F/F-aP2-Cre mice have increased insulin sensitivity. (A) Serum glucose values from control and knockout mice $(n=10-29)$. ${ }^{\star} P<0.00002$. (B) Serum insulin levels $(n=7-11)$. ${ }^{*} P<0.05$. (C) HOMA values $(n=4-6)$. ${ }^{*} P<0.05$. (D) Glucose tolerance test $(n=4-5)$. ${ }^{*} P<0.02$. Results are shown as mean + SEM or mean \pm SEM. $P$ values are as compared with control mice. (E) Immunoblots of total protein from WAT of control and knockout mice 30 minutes after insulin injection for phospho-Akt (p-Akt), total Akt, phospho-GSK-3 $\alpha / \beta$ (p-GSK-3 $\alpha / \beta)$, and total GSK-3 $\beta$.

relative to control mice (Supplemental Figure 4, A and B). Differences in body weight could not be explained by hypophagia, as daily food intake was similar in control and knockout mice on either diet (Supplemental Figure 4C).

Along with their decrease in total body weight, ATG7F/F-aP2-Cre mice fed either diet had a marked decrease in the size of gonadal WAT as determined visually (Figure 2C), by total WAT weight (Figure 2D), or by WAT weight normalized to total body weight (Figure $2 \mathrm{E})$. An opposite effect was seen in a fat depot composed primarily of brown adipocytes, as the weight of interscapular BAT was significantly increased in the knockout animals (Figure 2, F and G). HFDfed ATG7F/F mice had normal levels of serum leptin, whereas levels in knockout animals were significantly decreased in parallel with the reduction in fat mass (Supplemental Figure 5A). Serum adiponectin levels were normal in both control and null mice (Supplemental Figure 5B). In contrast to the marked differences in adipose weights, the absolute or relative weights of nonadipose organs including liver, heart, lung, kidney, and spleen were generally equivalent or only slightly decreased in knockout mice (Supplemental Figure 6, $A$ and $B)$, indicating a selective effect of the Atg7 knockout on adipose mass. Magnetic resonance spectroscopy of HFD-fed animals confirmed a $60 \%$ decrease in total fat mass in ATG7F/F-aP2-Cre mice (Figure $2 \mathrm{H}$ ) but only a 17\% decrease in lean mass (Figure 2I). Thus, the decrease in body weight in $\mathrm{ATG} 7^{\mathrm{F} / \mathrm{F}}$-aP2-Cre mice was predominantly secondary to a reduction in WAT mass.

$A T G 7^{F / F}$-aP2-Cre mice have improved insulin sensitivity and fail to accumulate TG in nonadipose tissues. Increased adiposity is associated with insulin resistance, and the decrease in WAT mass in ATG $7^{\mathrm{F} / \mathrm{F}}$-aP2-Cre mice suggested that these animals may be more insulin responsive. HFD-fed knockout mice had decreased serum glucose and insulin levels as compared with control mice (Figure 3 , A and B). As a result, homeostasis model of insulin resistance (HOMA) values were significantly reduced in null mice (Figure $3 \mathrm{C}$ ), indicating increased insulin sensitivity in these animals. Glucose tolerance tests revealed that HFD-fed knockout mice had significantly increased glucose clearance as compared with control mice (Figure 3D). Finally, activation of the insulin signaling pathway was greater in WAT from ATG7 $7^{\mathrm{F} / \mathrm{F}}$-aP2-Cre mice as determined by the relative increase in levels of phospho-Akt and phospho-glycogen synthase kinase 3 (phospho-GSK-3) after insulin injection (Figure 3E). Mice with an inhibition of adipocyte macroautophagy therefore maintained greater insulin sensitivity when challenged with an HFD.
Limited TG storage capacity in adipose tissue can lead to an overflow of fatty acids into the blood and deleterious lipid deposition in other organs. However, serum fatty acid levels were equivalent in control and knockout mice on RD or HFD (Supplemental Figure 7A). Serum TG and cholesterol levels were decreased in the null mice on either diet, but the differences were significant only for cholesterol levels in RD-fed animals (Supplemental Figure 7, B and C). To assess whether nonadipose tissue lipid accumulation resulted from reduced WAT mass in ATG7 ${ }^{\mathrm{F} / \mathrm{F}}$-aP2-Cre mice, hepatic and cardiac TG levels were determined. No TG accumulation occurred in the livers or hearts of knockout mice on either diet, and RD-fed null mice had an actual decrease in hepatic TG content (Supplemental Figure 8, A and $\mathrm{B})$. The reduction in WAT mass therefore had the beneficial effect of increasing insulin sensitivity and did not come at the cost of increased serum fatty acid levels or lipid deposition in nonadipose tissues.

WAT in ATG $7^{F / F}$-aP2-Cre mice has histological characteristics of BAT. Along with the dramatic reduction in WAT mass, prominent histological differences were apparent in the gonadal WAT from ATG7 ${ }^{\mathrm{F} / \mathrm{F}}$-aP2-Cre mice fed either RD or HFD (Figure $4 \mathrm{~A}$ ). First, the adipocytes in the WAT of the knockout mice were more variable in size but overall much smaller. Second, a significant number of adipocytes in WAT from knockout mice had smaller multilocular lipid droplets (LD) in contrast with the single large unilocular LD seen in the overwhelming majority of adipocytes from control mice. Finally, knockout adipocytes had greater cytoplasmic area not occupied by LD. Morphometric analysis confirmed these findings. The smaller adipocyte size in WAT from knockout mice resulted in a greater than 4-fold increase in the number of adipocytes per area of adipose tissue (Figure 4B). The histological differences in LD were confirmed by findings of greater numbers of LD per unit area, smaller mean LD area, and increased number of cells with multiloculated LD in the WAT of ATG7 $7^{\mathrm{F} / \mathrm{F}}$-aP2-Cre mice (Figure 4, C-E). These features are all characteristic of BAT (8), suggesting that the loss of Atg7 in WAT led to increased numbers of adipocytes with features of brown adipocytes.

Electron microscopy of gonadal WAT revealed striking ultrastructural differences between adipocytes from littermate control and ATG7 $7^{\mathrm{F} / \mathrm{F}}$-aP2-Cre mice (Supplemental Figure 9A). In contrast with the characteristic appearance of control mouse WAT in which most of the adipocytes had a low content of cytosolic organelles and an abundance of vacuolated structures in the limiting region 
A

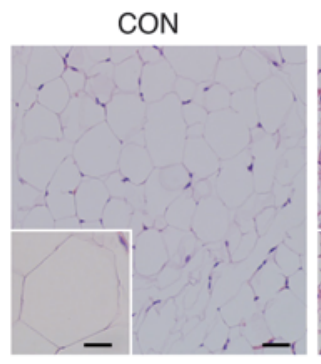

D

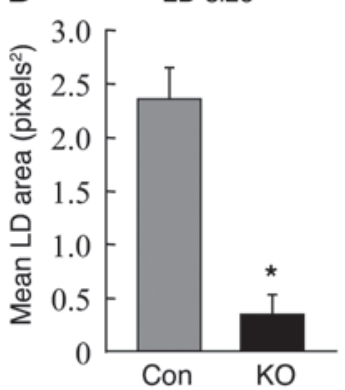

KO

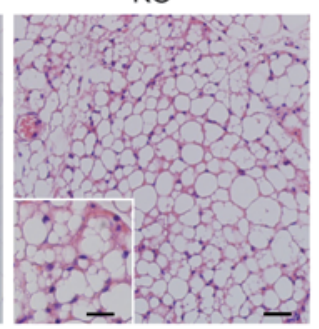

E

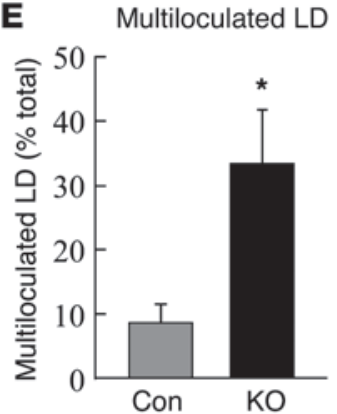

B

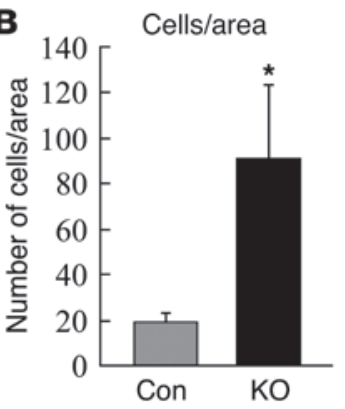

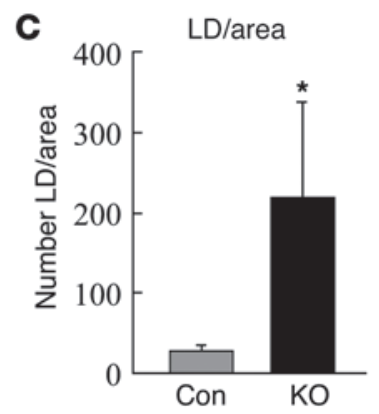

$\mathbf{F}$

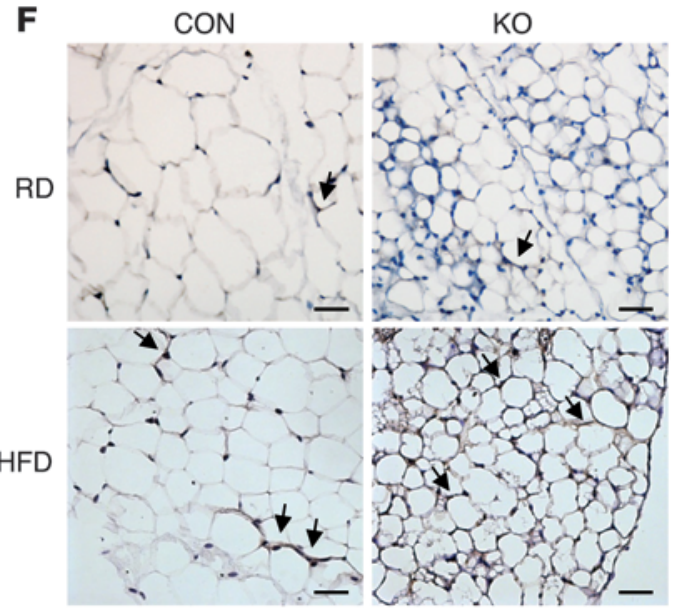

Figure 4

Gonadal WAT in knockout mice has characteristics of brown fat. (A) H\&E sections of WAT from control and knockout mice. Scale bars: 50 um; $20 \mu \mathrm{m}$ (insets). (B) Number of adipocytes per area. ${ }^{*} P<0.001$. (C) Number of LD per area. ${ }^{*} P<0.01$. (D) Mean LD area. ${ }^{*} P<0.000001$. (E) Percentage of adipocytes that have multiloculated LD. ${ }^{\star} P<0.00002$. Results are shown as mean + SEM $(n=5-6)$. $P$ values are as compared with control mice. (F) F4/80 immunohistochemical staining of control and knockout mice fed RD or HFD. Arrows indicate areas of positive staining for F4/80. Scale bars: $50 \mu \mathrm{m}$.

of the LD (Supplemental Figure 9A), cells in WAT from the knockout mice had a marked increase in intracellular organelles and lower levels of cytosolic vacuolation (Supplemental Figure 9A). Morphometric analysis of the mitochondrial content confirmed a significant increase in the total number of mitochondria and in the percentage of the cellular area occupied by these mitochondria in the WAT of ATG7 $7^{\mathrm{F} / \mathrm{F}}$-aP2-Cre mice as compared with that in control mice (Supplemental Figure 9, B and C). WAT from the knockout mice therefore exhibit both light and electron microscopic features of brown adipocytes.

Obesity is associated with a proinflammatory state in which adipose tissue becomes infiltrated with macrophages (16). To determine whether a loss of autophagy in adipocytes affected the macrophage inflammatory response to HFD, WAT from the 2 types of mice was examined by immunohistochemical staining for the macrophage-restricted cell-surface glycoprotein F4/80 (17). As expected, WAT from both $A T G 7^{\mathrm{F} / \mathrm{F}}$ and $\mathrm{ATG} 7^{\mathrm{F} / \mathrm{F}}$-aP2-Cre mice fed $\mathrm{RD}$ contained a few rare areas of cells staining positive for $\mathrm{F} 4 / 80$, indicating that significant macrophage infiltration did not occur in RD-fed animals irrespective of autophagic function (Figure 4F). With HFD feeding, sporadic positive staining was present in control mice (Figure 4F). In contrast, knockout mice fed the HFD had a marked increase in $\mathrm{F} 4 / 80$ staining (Figure $4 \mathrm{~F}$ ), suggesting that macroautophagy functions to prevent the development of HFDinduced adipose tissue macrophage infiltration.
Loss of autophagy alters expression of adipocyte differentiation factors in WAT. To further determine whether WAT from Atg7-knockout mice had molecular as well as histological characteristics of BAT, levels of proteins differentially expressed in the 2 forms of adipocytes were examined by immunoblotting. BAT is specialized for thermogenesis, which is mediated by uncoupling protein 1 (UCP-1), and UCP-1 expression is restricted to brown adipocytes (18). UCP-1 was essentially undetectable in WAT from RD- and HFD-fed control mice by immunoblotting, but high levels were present in WAT from ATG7 ${ }^{\mathrm{F} / \mathrm{F}}$-aP2-Cre mice (Figure $5 A)$. PPAR $\gamma$ coactivator $1 \alpha$ (PGC- $1 \alpha)$ regulates many of the differentiated features of brown fat, including UCP-1 expression and mitochondrial biogenesis (19). Low levels of PGC-1 $\alpha$ were present in control mouse WAT, and levels increased in knockout mice (Figure 5A). Consistent with elevated levels of PGC- $1 \alpha$ and electron microscopic findings of increased numbers of mitochondria, protein levels of the mitochondrial enzymes cytochrome oxidase and cytochrome $c$ were markedly elevated in knockout mice as compared with control animals (Figure 5A). The elevated levels of UCP-1, PGC-1 $\alpha$, cytochrome oxidase, and cytochrome $c$ in knockout mouse WAT were all intermediate between those in normal WAT and BAT (Figure 5B). Thus, in the absence of autophagy, significant numbers of the adipocytes in WAT acquired molecular as well as histological characteristics of brown adipocytes. 
A

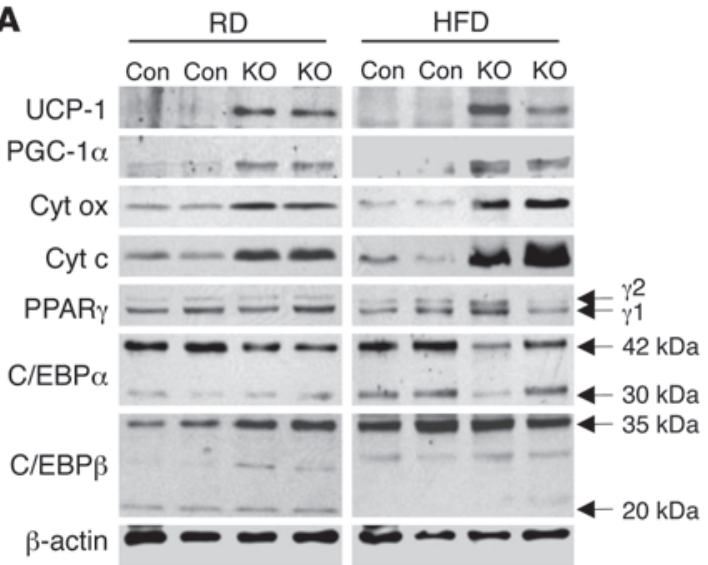

D

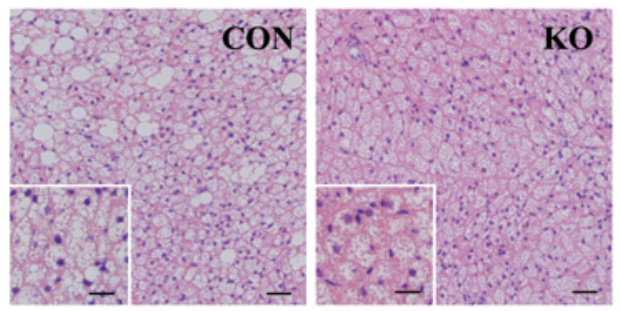

B

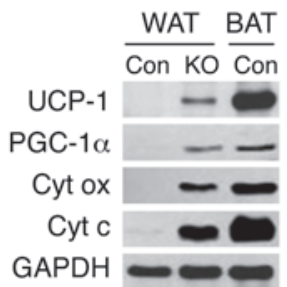

c

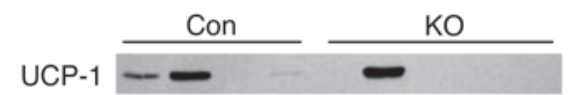

PGC- $1 \alpha$

Cyt ox

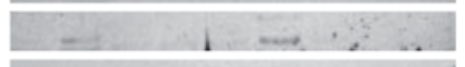

Cyt c

$\beta$-actin

E

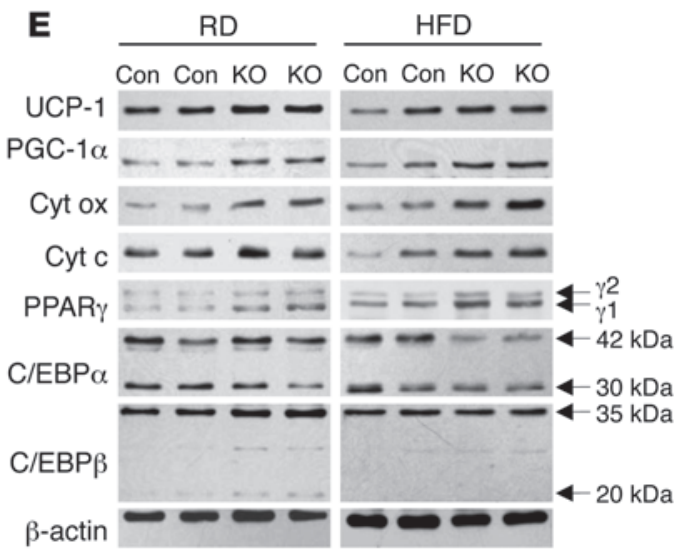

\section{Figure 5}

Loss of Atg7 alters the molecular characteristics of WAT and BAT. (A) Western blots of proteins from control and knockout mice fed RD or HFD. Cyt ox, cytochrome oxidase, cyt c, cytochrome c. (B) Western blots of protein samples from WAT and BAT of HFD-fed mice. (C) Immunoblots of protein isolates from WAT of young control and knockout mice. (D) Representative H\&E sections of interscapular BAT. Scale bars: 50 um; $20 \mu \mathrm{m}$ (insets). (E) Western blots of BAT protein from RD- and HFD-fed mice.

Other transcriptional regulators of differentiation common to both types of adipocytes were examined. Levels of PPAR $\gamma$, a critical mediator of both white and brown adipocyte differentiation (20), were variable among animals but overall equivalent in control and knockout mice (Figure 5A). C/EBP $\alpha$ levels were decreased in WAT from ATG7F/F-aP2-Cre mice, whereas C/EBP $\beta$ levels were unchanged (Figure 5A), consistent with increased levels of $\mathrm{C} / \mathrm{EBP} \beta$ relative to $\mathrm{C} / \mathrm{EBP} \alpha$ favoring decreased lipid storage and mitochondrial biogenesis (21).

Evidence of increased features of brown adipocytes in the WAT of 12-week-old ATG7F/F-aP2-Cre mice indicated that a loss of autophagy promoted either the differentiation of brown adipocyte precursors or the transdifferentiation of white adipocytes into brown adipocytes in fat pads that normally develop into WAT. To differentiate between these 2 possibilities, the gross phenotype and gonadal WAT of 3-week-old mice were examined. At 3 weeks of age, no significant differences were seen between control and null mice for total body, gonadal WAT, and interscapular BAT weights (Supplemental Figure 10, A-C). In contrast with the complete lack of UCP-1 expression in the WAT from older mice, UCP-1 was present in the WAT from several of the young littermate control mice, suggesting the presence of brown adipocytes that were subsequently lost with aging (Figure 5C). In contrast to the marked increase in UCP-1 levels in older knockout mice, ATG7F/F-aP2-Cre mice rarely had detectable UCP-1 at 3 weeks of age (Figure 5C). Levels of PGC-1 $\alpha$, cytochrome oxidase, and cytochrome $c$ mirrored those of UCP-1 and were also equivalent in control and null young mice (Figure $5 \mathrm{C})$. These findings indicate that the increase in brown adipocyte features in WAT developed after initial WAT formation.

$A T G 7^{F / F}$-aP2-Cre mouse WAT contains normal numbers of progenitor cells. Although significant differences in WAT differentiation were detected with the loss of macroautophagy, the possibility remained that a reduced white adipocyte proliferative capacity also contributed to the decrease in WAT in knockout mice. A subpopulation of the adipose stromal vascular cells that are Lin ${ }^{-}$ , $\mathrm{CD} 29^{+}, \mathrm{CD} 34^{+}, \mathrm{Sca}-1^{+}$, and $\mathrm{CD} 24^{+}$has been identified recently as WAT progenitor cells (22). As aP2 is not expressed in these undifferentiated cells, they should not undergo Atg7 excision or have impaired autophagic function. However, it is possible that secondary changes may have occurred in these progenitor cells as the result of altered adipocyte differentiation and/or loss of adipocyte mass. An examination of this cell subpopulation revealed that the relative percentage of progenitor cells in control mice was similar to that previously reported (22) and 
A

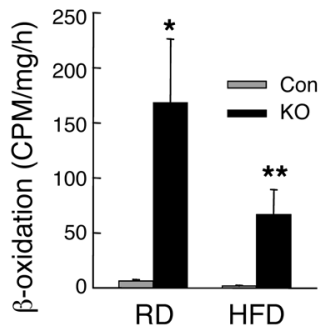

B

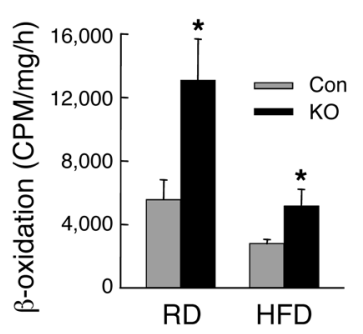

C

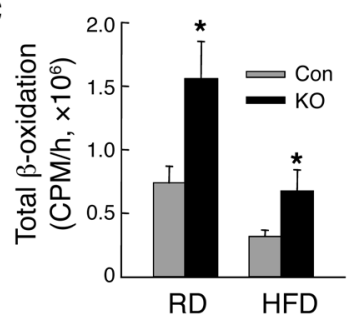

Figure 6

Levels of fatty acid $\beta$-oxidation are increased in Atg7-knockout mice. (A) Rates of gonadal WAT $\beta$-oxidation in control and knockout mice fed RD or HFD $(n=3-12)$. ${ }^{*} P<0.05$; ${ }^{* *} P<0.001$. (B) Rates of $\beta$-oxidation in interscapular BAT $(n=3-10)$. ${ }^{*} P<0.02$. (C) Total rate of WAT and BAT $\beta$-oxidation $(n=3-11) .{ }^{*} P<0.05$. Results are shown as mean + SEM. $P$ values are as compared with control mice fed the same diet.

equivalent to that in ATG7F/F-aP2-Cre mice (Supplemental Figure 11). The loss of autophagy therefore had no effect on the number of WAT progenitor cells present in the adipose tissue stromal vascular compartment.

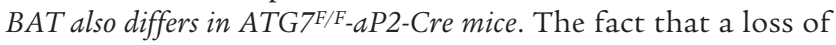
adipocyte autophagy induced changes in BAT as well as WAT was suggested by findings of increased interscapular BAT weight in the knockout animals (Figure 2, F and G). Histology of interscapular BAT from RD- and HFD-fed null mice was remarkable for an absence of large LD-containing cells and the overall presence of fewer and smaller-sized LD (Figure 5D). BAT immunoblots revealed slight increases in UCP-1, PGC-1 $\alpha$, and the mitochondrial enzymes cytochrome oxidase and cytochrome $c$ in RD- and HFD-fed knockout animals (Figure 5E). A slight increase in PPAR $\gamma$ and decrease in $\mathrm{C} / \mathrm{EBP} \alpha$ levels also occurred in knockout mouse fat. Thus, changes in BAT in animals lacking autophagy were consistent with those found in WAT but less significant because they occurred in the background of a fat depot already composed of brown adipocytes.

Adipocytes from $A T G 7^{F / F}$-aP2-Cre mice have increased levels of fatty acid $\beta$-oxidation. A metabolic characteristic of brown adipocytes is their higher rate of fatty acid $\beta$-oxidation that performs the beneficial function of expending excess energy. The lean body composition in the setting of an increased brown adipocyte mass in the knockout animals could therefore reflect greater rates of fatty acid $\beta$-oxidation in the WAT and BAT of ATG7-aP2-Cre mice. Rates of $\beta$-oxidation were increased in gonadal WAT from RD- and HFDfed ATG7F/F-aP2-Cre mice by 28- and 33-fold, respectively (Figure $6 \mathrm{~A})$. Compatible with the more modest histological and molecular changes found in BAT from knockout mice, the rate of $\beta$-oxidation was increased 2-fold in the interscapular BAT of these animals on either diet (Figure 6B).

To better assess the global effect of a knockout of autophagy on fatty acid $\beta$-oxidation, the total amount of $\beta$-oxidation was calculated for gonadal WAT and interscapular BAT taking into account the fat pad mass of each animal. The total level of $\beta$-oxidation was increased 2-fold in ATG7 ${ }^{\mathrm{F} / \mathrm{F}}$-aP2-Cre mice fed either diet (Figure $6 \mathrm{C})$. This effect essentially reflected the differences between $\beta$-oxidation rates in BAT, which had a substantially higher rate than WAT even in the knockout animals. Thus, the loss of autophagy in adipose tissue markedly increased levels of $\beta$-oxidation, which contributed to the decrease in body weight and WAT mass and accounted for the lack of an increase in serum fatty acid levels or lipid deposition in nonadipose organs in the knockout animals. with an increase in the mass of normal BAT led to a significantly increased rate of fatty acid $\beta$-oxidation. In vivo, these effects of a loss of autophagy in adipocytes translated into a leaner animal with increased insulin sensitivity. These findings demonstrate that the function of autophagy in the regulation of the differentiated state and metabolic function of adipocytes is of significant magnitude to alter whole-body energy homeostasis.

In 3T3-L1 cells, autophagic function was required for these cells to differentiate into white adipocytes. In the more complex in vivo situation of the mouse model with an inhibition of autophagy in adipose tissue, 2 striking changes occurred in WAT. First, WAT mass decreased dramatically, indicating that a loss of autophagy led to a reduction in adipocyte size and/or cell number. The 4 -fold increase in the number of cells per tissue area, along with the marked reduction in LD size, indicates that the predominant effect was a decrease in cell size. Decreased cell size presumably resulted from the marked reduction in stored lipid in these cells, as the intracellular space of a mature white adipocyte is composed almost entirely of a single large LD. In addition to the decrease in mass, the second effect in WAT in the Atg7-knockout mice was the presence of features of brown fat, as indicated by characteristic histology, increased number of mitochondria, and elevated levels of molecular markers of brown adipocytes such as UCP-1, PGC- $1 \alpha$, and mitochondrial enzymes. Thus, 2 possible mechanisms exist for the decrease in lipid storage in WAT. First, a block in white adipocyte differentiation may have led to a failure of these cells to accumulate lipid, without which they remained small in size. Alternatively, the increase in fatty acid $\beta$-oxidation and uncoupled respiration that occurred with the conversion of WAT to brown adipocytes, together with the increase in BAT mass, may have decreased the amount of lipid available for storage in WAT. The absence of any increase in serum fatty acids and the failure to accumulate excess lipid in nonadipose organs such as liver and heart that would occur if WAT lipid storage was simply blocked without any increase in energy usage suggest that increased energy expenditure was the main mechanism for the decrease in WAT mass.

Macroautophagy may regulate the balance between white and brown adipocytes in WAT by 1 of 2 mechanisms. First, autophagy may be critical for white adipocyte differentiation from a progenitor cell, and in the absence of autophagic function, these adipocyte precursors may be shunted into a default pathway of brown adipocyte differentiation. Against this possibility is the current evidence that white and brown adipocytes develop from distinct rather than common precursor cells $(10,11)$. In addition, the adipocyte-specific 
cre promoter aP2 used in these studies should not be expressed in undifferentiated progenitor cells. Therefore, these cells should not experience a loss of autophagic function. As expected, studies of the white adipocyte progenitor cell population therefore revealed no change in the number of these cells in the knockout mice. Finally, very young mice failed to exhibit the phenotypic or WAT changes seen in older mice, indicating that the effect of a loss of autophagy occurred after WAT formation. The second, more likely possibility is therefore that loss of autophagy altered adipocyte transdifferentiation by promoting the conversion of WAT into BAT or blocking BAT transdifferentiation to WAT. Supporting the former possibility is that it is known that WAT can acquire features of brown fat (23). PGC- $1 \alpha$ is a critical mediator of brown fat differentiation (24), and exogenous PGC- $1 \alpha$ overexpression induces the development of features of brown fat in WAT (19). PGC-1 $\alpha$ was induced in the WAT of Atg7-knockout mice, indicating that in the absence of autophagy, increased numbers of cells had entered a brown adipocyte differentiation pathway. In rodents, BAT develops prenatally and WAT postnatally (8), and BAT conversion to WAT may contribute to its formation. In larger mammals, including humans, BAT is partially replaced after birth with WAT, a process that may occur through transdifferentiation (25). Autophagy could mediate adipocyte transdifferentiation through several mechanisms. Autophagy may be critical for the cytoplasmic remodeling necessary for transdifferentiation of BAT to lipid-storing WAT such as through removal of the excessive mitochondria present in brown adipocytes. Alternatively, autophagy may promote the removal and degradation of a protein or proteins critical for the maintenance of the brown adipocyte phenotype.

An additional finding in the WAT from HFD-fed knockout mice was an increase in macrophage infiltration. Adipose tissue infiltration with macrophages is a known feature of obesity (16), although the mechanism of this inflammatory response is unclear. This increase in macrophage infiltration in knockout animals could not explain their changes in WAT because increased numbers of macrophages were seen only in HFD-fed mice and the effect of the loss of autophagy on WAT mass and adipocyte differentiation was equivalent in RD- and HFD-fed animals. In addition, a loss of macroautophagy was sufficient by itself to block the differentiation of cultured preadipocytes in vitro despite an absence of macrophages. The fact that the increase in macrophage infiltration was restricted to HFD-fed animals suggests that the typical development of adipose tissue inflammation in response to lipid excess was somehow accelerated by the absence of adipocyte autophagic function. Interestingly, some studies have associated macrophage infiltration with dying adipocytes in what have been termed crown-like structures $(26,27)$. Given the known protective function of autophagy against cell death (28), it is possible that the defect in autophagy sensitized white adipocytes to death from factors related to the presence of excessive lipid.

The overall effect of the adipocyte-specific loss of macroautophagy was a lean mouse with improved insulin sensitivity secondary to a reduction in WAT mass resulting from increased energy expenditure by brown adipocytes. Consistent with this conclusion are studies of other transgenic mice characterized by increased brown adipocytes in WAT that also had a lean phenotype, increased insulin sensitivity, and protection from diet-induced obesity $(29,30)$. Increased numbers of brown adipocytes with their higher mitochondrial content, ability to uncouple cellular respiration through
UCP-1, and increased fatty acid breakdown by $\beta$-oxidation led to increased energy usage. This conclusion is consistent with prior studies of UCP-1 overexpression in WAT in which increased fatty acid oxidation occurred and enhanced whole-body glucose tolerance (31). The marked decrease in WAT mass in the knockout mice could have had the detrimental effect of increasing the delivery and sequestration of lipid in nonadipose organs such as liver and heart secondary to the reduction in adipose tissue capacity to store lipid. This fat deposition in nonadipose organs failed to occur in ATG7 ${ }^{\mathrm{F} / \mathrm{F}}$-aP2-Cre mice because excess lipid was oxidized and did not require storage. Loss of normal WAT depots did lead to some peri- and postnatal mortality. Although the cause of this mortality remains to be identified, it may have resulted from insufficient lipid storage to meet the energy demands of acute stresses.

The present findings are in direct opposition to those from previous studies in which a pharmacological or genetic inhibition of the mTOR pathway was examined in adipocytes. Autophagy is blocked by mTOR, and its inhibition should therefore increase autophagic function. Studies of 3T3-L1 cells demonstrated that treatment with the mTOR inhibitor rapamycin blocked adipogenic differentiation $(32,33)$. Recent investigations of mice with an adipocyte-specific knockout of the mTOR component raptor produced a phenotype similar to our findings in ATG $7{ }^{\mathrm{F} / \mathrm{F}}$-aP2-Cre mice - a lean mouse with decreased adipose tissue and increased metabolic activity (34). The fact that a specific genetic inhibition of autophagy yielded findings similar to those in studies in which autophagy was presumably augmented by inhibition of the mTOR pathway indicates that the reported effects of blocking mTOR in adipocytes must result from one of the many mTOR-dependent pathways other than autophagy. This speculation is strongly supported by the fact that a knockdown of either of 2 distinct autophagy genes, Atg7 or Atg5, led to the identical finding of an inhibition of 3T3-L1 preadipocyte differentiation.

The effect of a loss of autophagy on adipose tissue lipid storage differs from that which we have recently reported for a hepatocyte-specific ablation of macroautophagy in the liver (35). In the liver, an inhibition of autophagy had the net metabolic effect of promoting hepatocyte lipid accumulation because autophagy was critical for the lipolysis of TG stored in LD (35). It is not surprising that hepatocytes have mechanisms of lipid breakdown that are absent in adipocytes, which contain greater amounts of lipolytic enzymes, such as hormone-sensitive lipase. Initially the existence of opposing functions for autophagy in the 2 primary lipid storage organs in the body seems counterproductive, promoting lipid accumulation in adipose tissue but breaking down stored fat in the liver. However, these actions may be viewed as complementary. An intact autophagic system could serve to prevent lipotoxicity in a nonadipose organ such as the liver by both preventing hepatic lipid retention and promoting safer storage in adipose tissue. It remains to be seen whether an impairment in autophagy that commences later in life, as occurs in humans with aging, rather than early in life, as was studied in these 2 rodent models, has similar or different effects on adipose tissue and liver lipid metabolism.

The current findings demonstrate a major function for autophagy in the regulation of adipocyte mass and differentiation and provide new insight into the mechanisms regulating whether adipose tissue is composed of white or brown adipocytes. Recent studies have indicated that the amount of BAT in humans is inversely correlated with body mass $(2,3)$, suggesting that BAT 
size may regulate body weight. The ability of an inhibition of adipocyte macroautophagy to increase the quantity of brown fat may have important implications for developing a novel therapeutic approach to improving energy homeostasis in human conditions such as obesity and the metabolic syndrome.

\section{Methods}

Cells and culture conditions. 3T3-L1 cells were maintained in DMEM (Mediatech) supplemented with $10 \%$ FBS (Gemini) and antibiotics (Invitrogen) at $37^{\circ} \mathrm{C}$ on plastic dishes (Falcon; BD Biosciences). Adipocyte differentiation was initiated by culturing the cells in maintenance medium supplemented with $160 \mathrm{nM}$ insulin, $250 \mathrm{nM}$ dexamethasone, and $0.5 \mathrm{mM}$ 1-methyl-3isobutylxanthine (day 0). Forty-eight hours later, the cells were switched to maintenance medium supplemented just with insulin. After 48 hours in this medium, the cells were placed back in unsupplemented maintenance medium, which was changed every 48 hours. Some cells were treated on day 0 with $10 \mathrm{mM}$ 3-methyladenine (Sigma-Aldrich), $20 \mathrm{mM}$ ammonium chloride, or $100 \mu \mathrm{M}$ leupeptin (Fisher).

Lentiviral shRNA construction. Oligonucleotides with the following nucleotide sequences were used for the cloning of shRNA-encoding sequences into a lentiviral vector: ATG7: 5'-GATCCCCGCAGCTCATTGATAACCATTTCAAGAGAATGGTTATCAATGAGCTGCTTTTTC-3'; ATG5 no. 1: 5'-GATCCCCGTGATCAACGAAATGCAGATTCAAGAGATCTGCATTTCGTTGATCACTTTTTGGAAA-3'; and ATG5 no. 2: GATCCCССТTTCTTCATATTAGCATTTCAAGAGAAATGCTAATATGAAGAAAGTTTTTGGAAA. The oligonucleotides were annealed and cloned into pSUPER (Ambion) at the BglII-XhoI site. The SmaI-XhoI fragments of the corresponding PSUPER plasmids, which included the H1 promoter-shRNA cassette, were subcloned into the EcoRV-XhoI sites of the vector PCCL.sin.PPT.hPGK.GFPWpre (36).

High-titer lentiviral stocks were produced by calcium phosphate-mediated transfection of the modified transfer vectors and the packaging vectors pMDLg/pRRE, pRSV-Rev, and pMD2.VSVG into HEK-293T cells. Supernatants were harvested over 36 to 48 hours, titered by plaque assay, and used at an MOI of 5 to infect 3T3-L1 cells. The efficiency of infection was determined at 72 hours by the number of GFP-positive cells under fluorescence microscopy that exceeded $98 \%$. A polyclonal cell line transfected with the vector lacking any shRNA (VEC cells) was also established and served as a control.

TG assays. Cellular and tissue TG content was determined by the Trig/GB Kit (Roche), as previously described (37). Serum TG levels were measured by the Cayman TG kit.

Fluorescence microscopy. The numbers of apoptotic and necrotic cells were quantified by fluorescence microscopy after costaining with acridine orange and ethidium bromide, as described previously (38). A minimum of 400 cells/dish were examined for morphometric changes of apoptosis or positive acridine orange staining indicative of necrosis.

Animal models. To generate mice with an adipocyte-specific knockout of macroautophagy, ATG7 $7^{\mathrm{F} / \mathrm{F}}$ mice (provided by M. Komatsu and K. Tanaka, Tokyo Metropolitan Institute of Medical Science, Tokyo, Japan; ref. 39) were crossed with mice expressing Cre recombinase under the control of the adipocyte-specific promoter aP2 (Jackson Laboratory) (40). Studies were performed in $\mathrm{ATG} 7^{\mathrm{F} / \mathrm{F}}$-aP2-Cre mice and littermate controls lacking the cre transgene (ATG7 $7^{\mathrm{F} / \mathrm{F}}$ mice). Mice were maintained under 12-hour light/12-hour dark cycles with unlimited access to food and water. Genotyping was performed by PCR with established primers $(39,40)$. After weaning, mice were started on RD (no. 5058; LabDiet) or HFD (60\% kcal in fat; no. D12492, Research Diets). All studies were approved by the Animal Care and Use Committee of the Albert Einstein College of Medicine and followed the NIH guidelines on the care and use of animals.
Magnetic resonance spectroscopy. Body composition was determined by using an ECHO magnetic resonance spectroscopy instrument (Echo Medical Systems).

Serum assays. Blood glucose values were determined with an Ascensia Contour glucose meter (Bayer HealthCare). Serum insulin levels were measured by radioimmunoassay, as previously described (41). Levels of insulin resistance were determined by HOMA (insulin resistance $=[$ fasting glucose $(\mathrm{mmol} / \mathrm{l}) \times$ fasting insulin $(\mu \mathrm{U} / \mathrm{ml})] / 22.5)(42)$. Serum leptin and adiponectin levels were determined by ELISA (R\&D Systems). Serum-free fatty acid and cholesterol levels were measured by commercial kits from Wako and Invitrogen, respectively. Studies were performed in fed mice.

Glucose tolerance test. Following an overnight fast, mice received an intraperitoneal injection of glucose ( $2 \mathrm{mg} / \mathrm{g}$ body weight). Blood glucose levels were determined at baseline and over time as described above.

Insulin injection. Mice were injected with 0.75 units $/ \mathrm{kg}$ of insulin intraperitoneally and their gonadal WAT removed 30 minutes later for Western blot analysis.

Protein isolation and Western blot. Total protein was isolated from cells harvested and centrifuged in PBS, pH 7.5. The pellets were resuspended and sonicated in buffer containing $20 \mathrm{mM}$ Tris, $\mathrm{pH} 7.5,1 \%$ Triton X-100, $1 \mathrm{mM}$ EDTA, $1 \mathrm{mM}$ EGTA, and protease and phosphatase inhibitors as described previously (43). The lysates were subjected to Western blot analysis. Total protein from adipose tissue was isolated by homogenization in cold RIPA buffer that contained $50 \mathrm{mM}$ Tris, pH 7.5, $150 \mathrm{mM}$ sodium chloride, $1 \%$ NP-40, $0.5 \%$ sodium deoxycholate, $0.1 \%$ SDS, $0.1 \mathrm{mM}$ EDTA, $0.1 \mathrm{mM}$ EGTA, and the appropriate protease and phosphatase inhibitors. The lysates were centrifuged and the supernatants used for Western blotting. Protein concentrations were estimated using the Bio-Rad protein assay according to the manufacturer's instructions.

Western blotting was performed by denaturing $50 \mu \mathrm{g}$ of protein at $100^{\circ} \mathrm{C}$ for 5 minutes in Laemmli sample buffer containing $62.5 \mathrm{mM}$ Tris, $\mathrm{pH} 6.8$, $2 \%$ SDS, $25 \%$ glycerol, $0.01 \%$ bromophenol blue, and $5 \% \beta$-mercaptoethanol. Samples were subjected to SDS-polyacrylamide gel electrophoresis and resolved at $100 \mathrm{~V}$ over 3 hours. Proteins were transferred to a nitrocellulose membrane (Schleicher \& Schuell) in transfer buffer containing $25 \mathrm{mM}$ Tris, $\mathrm{pH}$ 8.3, $192 \mathrm{mM}$ glycine, $0.01 \%$ SDS, and 15\% methanol using a Bio-Rad Trans-blot SD semidry transfer cell to which $150 \mathrm{~mA}$ were applied for 90 minutes. Membranes were blocked in 5\% nonfat dry milk, $20 \mathrm{mM}$ Tris, pH 7.5, $500 \mathrm{mM}$ sodium chloride, and 0.5\% Tween-20 (TBS-T) for 1 hour. Membranes were exposed to antibodies that recognized $\mathrm{C} / \mathrm{EBP} \alpha, \mathrm{C} / \mathrm{EBP} \beta, \mathrm{PGC}-1 \alpha$, and aP2 (Santa Cruz Biotechnology Inc.); Atg7, LC3, PPAR $\gamma$, phospho-Akt, Akt, phospho-GSK-3 $\alpha / \beta$, GSK-3 $\beta$, and SCD1 (Cell Signaling Technology); UCP-1 (Abcam); cytochrome $c$ and FAS (BD Biosciences); Atg5 (Novus); and cytochrome oxidase (Mitosciences). The GLUT4 antibody was a gift from M. Charron (Albert Einstein College of Medicine). Equivalent protein loading between the samples was verified by reprobing membranes for $\beta$-actin or glyceraldehyde 3 -phosphate dehydrogenase (Abcam). Primary antibodies were used at 1:1000 to 1:3000 dilutions in $5 \%$ BSA or nonfat milk for 18 hours at $4{ }^{\circ} \mathrm{C}$. Membranes were exposed to anti-rabbit, anti-mouse, or anti-goat secondary antibodies conjugated with horseradish peroxidase (KPL) at a dilution of 1:10,000 in 5\% nonfat milk TBS-T for 1 hour at room temperature. Signals were detected with a chemiluminescence detection system (Western Lightning Chemiluminescence Plus; PerkinElmer Life Sciences,) and exposure to x-ray film.

Histological analysis. Morphometric analysis of adipocytes was performed on H\&E sections using NIH ImageJ software (http://rsbweb.nih. gov/ij/). Perimeters of the individual LD, identified by their characteristic homogenous density, were delineated with the free-hand selection tool, and the mean perimeter value of at least 25 droplets per section was determined. The total number of $\mathrm{LD}$ was quantified with the analyzed 
particle tool and then normalized to the number of adipocyte nuclei per section. LD were scored as containing single or multiple droplets by visual counting of individual cells.

Immunohistochemistry. Five-micrometer paraffin sections were treated with proteinase $\mathrm{K}$ for antigen retrieval. Sections were stained with an F4/80 antibody using the BioModule Immunohistochemical Staining Kit (Invitrogen).

Analysis of adipose stromal vascular cells. The population of adipocyte progenitor cells in WAT designated $\mathrm{Lin}^{-}, \mathrm{CD} 29^{+}, \mathrm{CD} 34^{+}, \mathrm{Sca}-1^{+}$, and CD24+ was identified by FACS analysis, as previously reported (22). Adipose tissue was harvested and the stromal vascular cells isolated by enzymatic digestion (Liberase Blendzyme 3; Roche). The digested tissue was centrifuged to remove adipocytes and released lipids and then incubated with an ammonium chloride lysis buffer to remove red blood cells. The isolated stromal vascular cells were resuspended in cold PBS with $2 \% \mathrm{FBS}$ and $0.1 \%$ sodium azide for labeling. Samples were blocked with anti-CD16/32 (eBioscience) for 10 minutes at $4^{\circ} \mathrm{C}$. They were then labeled for 30 minutes at $4^{\circ} \mathrm{C}$ with anti-CD45-PE-Cy7, anti-CD31-PE-Cy7, anti-CD34-FITC, anti-Ter119PE-Cy7, anti-Sca1-PE-Cy5, anti-CD29-APC, and anti-CD24-PE (eBioscience). Samples were fixed in $1 \%$ formalin and analyzed on a BD LSRII flow cytometer (BD Biosciences) using FlowJo (Treestar) software. Cells not expressing CD45, CD31, or Ter119 were considered Lin-

Fatty acid $\beta$-oxidation. The rate of adipose tissue fatty acid $\beta$-oxidation was determined by modification of the method described by Dohm et al. (44). Freshly isolated gonadal WAT or interscapular BAT (50-100 mg) was gently homogenized in ice-cold buffer containing $130 \mathrm{mM} \mathrm{NaCl}, 47 \mathrm{mM} \mathrm{KCl}$, $1.2 \mathrm{mM} \mathrm{KH}_{2} \mathrm{PO}_{4}, 10 \mathrm{mM}$ HEPES, $1 \mathrm{mM} \mathrm{CaCl}_{2}, 1.2 \mathrm{mM} \mathrm{MgCl}_{2}$, and $2 \mathrm{mM}$ sodium pyruvate at $\mathrm{pH} 7.4$ and the homogenate diluted 5 -fold (w/v) with cold buffer. The rate of $1{ }^{14} \mathrm{C}$-oleic acid oxidation was determined by measuring the amount of ${ }^{14} \mathrm{CO}_{2}$ released over 1 hour. In brief, $0.5 \mathrm{ml}$ of the diluted homogenate was mixed with $2 \mathrm{ml}$ of a reaction mixture that contained $2 \mathrm{mM}$ ATP, $1 \mathrm{mM}$ L-carnitine, $0.5 \mathrm{mM}$ coenzyme A, $1 \mathrm{mM}$ dithiothreitol, $1 \mathrm{mM} \mathrm{MgCl}_{2}, 0.1 \mathrm{mM} \mathrm{NAD}^{+}, 100 \mathrm{mM}$ sucrose, $10 \mathrm{mM} \mathrm{KH}_{2} \mathrm{PO}_{4}$, $80 \mathrm{mM} \mathrm{KCl}$, and $0.1 \mathrm{mM}$ EDTA at $\mathrm{pH}$ 7.3. The reaction was initiated by the addition of $1-{ }^{14} \mathrm{C}$-oleic acid to the mixture, and ${ }^{14} \mathrm{CO}_{2}$ was trapped onto $\mathrm{NaOH}$-soaked filter paper. The rate of $\beta$-oxidation was expressed as the amount of ${ }^{14} \mathrm{CO}_{2}$ released per mg of tissue per hour. The total rate was also determined for the total mass of gonadal WAT and interscapular BAT.

Statistics. All numerical results are reported as mean + SEM or mean \pm SEM and represent data from a minimum of 3 independent experiments. Groups were compared by 2 -tailed Student's $t$ test. Statistical significance was defined as $P<0.05$. Calculations were made with Sigma Plot (Jandel Scientific).

\section{Acknowledgments}

This work was supported in part by NIH grants DK061498 (to M.J. Czaja), AG031782 (to A.M. Cuervo and M.J. Czaja), AG021904 (to A.M. Cuervo), DK020541 (to J.E. Pessin), DK047208 (to G.J. Schwartz), and DK020451 (to G.J. Schwartz). We thank Masaaki Komatsu and Keiji Tanaka for providing the ATG7F/F mice, Bert Kadereit and David L. Silver for their advice on the 3T3-L1 cell studies, Kathryn E. Tanaka for help with the histology, and Maureen Charron for providing the GLUT4 antibody.

Received for publication March 17, 2009, and accepted in revised form August 26, 2009.

Address correspondence to: Mark J. Czaja, Marion Bessin Liver Research Center, Albert Einstein College of Medicine, 1300 Morris Park Avenue, New York, New York 10461, USA. Phone: (718) 430-4255; Fax: (718) 430-8975; E-mail: mark.czaja@einstein.yu.edu.
1. Spiegelman, B.M., and Flier, J.S. 2001. Obesity and the regulation of energy balance. Cell. 104:531-543.

2. Cypess, A.M., et al. 2009. Identification and importance of brown adipose tissue in adult humans. N. Engl. J. Med. 360:1509-1517.

3. Marken Lichtenbelt, W.D., et al. 2009. Cold-activated brown adipose tissue in healthy men. $N$. Engl. J. Med. 360:1500-1508.

4. Nedergaard, J., Bengtsson, T., and Cannon, B. 2007. Unexpected evidence for active brown adipose tissue in adult humans. Am. J. Physiol. Endocrinol. Metab. 293:E444-E452.

5. Virtanen, K.A., et al. 2009. Functional brown adipose tissue in healthy adults. N. Engl. J. Med. 360:1518-1525.

6. Gesta, S., Tseng, Y.H., and Kahn, C.R. 2007. Developmental origin of fat: tracking obesity to its source. Cell. 131:242-256.

7. Tiraby, C., and Langin, D. 2003. Conversion from white to brown adipocytes: a strategy for the control of fat mass? Trends Endocrinol. Metab. 14:439-441.

8. Hansen, J.B., and Kristiansen, K. 2006. Regulatory circuits controlling white versus brown adipocyte differentiation. Biochem. J. 398:153-168.

9. Spalding, K.L., et al. 2008. Dynamics of fat cell turnover in humans. Nature. 453:783-787.

10. Seale, P., et al. 2008. PRDM16 controls a brown fat/ skeletal muscle switch. Nature. 454:961-967.

11. Timmons, J.A., et al. 2007. Myogenic gene expression signature establishes that brown and white adipocytes originate from distinct cell lineages. Proc. Natl. Acad. Sci. U. S. A. 104:4401-4406.

12. Cecconi, F., and Levine, B. 2008. The role of autophagy in mammalian development: cell makeover rather than cell death. Dev. Cell. 15:344-357.

13. Mizushima, N., and Yoshimori, T. 2007. How to interpret LC3 immunoblotting. Autophagy. 3:542-545.

14. Bernlohr, D.A., Angus, C.W., Lane, M.D., Bola- nowski, M.A., and Kelly, T.J., Jr. 1984. Expression of specific mRNAs during adipose differentiation: identification of an mRNA encoding a homologue of myelin P2 protein. Proc. Natl. Acad. Sci. U. S. A. 81:5468-5472.

15. Blommaart, E.F., Krause, U., Schellens, J.P., Vreeling-Sindelarova, H., and Meijer, A.J. 1997. The phosphatidylinositol 3-kinase inhibitors wortmannin and LY294002 inhibit autophagy in isolated rat hepatocytes. Eur. J. Biochem. 243:240-246.

16. Weisberg, S.P., et al. 2003. Obesity is associated with macrophage accumulation in adipose tissue. J. Clin. Invest. 112:1796-1808.

17. Austyn, J.M., and Gordon, S. 1981. F4/80, a monoclonal antibody directed specifically against the mouse macrophage. Eur. J. Immunol. 11:805-815.

18. Unami, A., Shinohara, Y., Kajimoto, K., and Baba, Y. 2004. Comparison of gene expression profiles between white and brown adipose tissues of rat by microarray analysis. Biochem. Pharmacol. 67:555-564.

19. Puigserver, P., et al. 1998. A cold-inducible coactivator of nuclear receptors linked to adaptive thermogenesis. Cell. 92:829-839.

20. Farmer, S.R. 2006. Transcriptional control of adipocyte formation. Cell Metab. 4:263-273.

21. Chiu, C.H., Lin, W.D., Huang, S.Y., and Lee, Y.H. 2004. Effect of a C/EBP gene replacement on mitochondrial biogenesis in fat cells. Genes Dev. 18:1970-1975.

22. Rodeheffer, M.S., Birsoy, K., and Friedman, J.M. 2008. Identification of white adipocyte progenitor cells in vivo. Cell. 135:240-249.

23. Tiraby, C., et al. 2003. Acquirement of brown fat cell features by human white adipocytes. J. Biol. Chem. 278:33370-33376.

24. Puigserver, P., and Spiegelman, B.M. 2003. Peroxisome proliferator-activated receptor- $\gamma$ coactivator 1 $\alpha$ (PGC-1 $\alpha)$ : transcriptional coactivator and meta- bolic regulator. Endocr. Rev. 24:78-90.

25. Lean, M.E., James, W.P., Jennings, G., and Trayhurn, P. 1986. Brown adipose tissue uncoupling protein content in human infants, children and adults. Clin. Sci. (Lond.). 71:291-297.

26. Cinti, S., et al. 2005. Adipocyte death defines macrophage localization and function in adipose tissue of obese mice and humans. J. Lipid Res. 46:2347-2355.

27. Murano, I., et al. 2008. Dead adipocytes, detected as crown-like structures, are prevalent in visceral fat depots of genetically obese mice. J. Lipid Res. 49:1562-1568.

28. Wang, Y., et al. 2008. Loss of macroautophagy promotes or prevents fibroblast apoptosis depending on the death stimulus. J. Biol. Chem. 283:4766-4777.

29. Cederberg, A., et al. 2001. FOXC2 is a winged helix gene that counteracts obesity, hypertriglyceridemia, and diet-induced insulin resistance. Cell. 106:563-573.

30. Tsukiyama-Kohara, K., et al. 2001. Adipose tissue reduction in mice lacking the translational inhibitor 4E-BP1. Nat. Med. 7:1128-1132.

31. Yamada, T., et al. 2006. Signals from intra-abdominal fat modulate insulin and leptin sensitivity through different mechanisms: neuronal involvement in food-intake regulation. Cell Metab. 3:223-229.

32. Bell, A., Grunder, L., and Sorisky, A. 2000. Rapamycin inhibits human adipocyte differentiation in primary culture. Obes. Res. 8:249-254.

33. Yeh, W.C., Bierer, B.E., and McKnight, S.L. 1995. Rapamycin inhibits clonal expansion and adipogenic differentiation of 3T3-L1 cells. Proc. Natl. Acad. Sci. U. S. A. 92:11086-11090.

34. Polak, P., et al. 2008. Adipose-specific knockout of raptor results in lean mice with enhanced mitochondrial respiration. Cell Metab. 8:399-410. 
35. Singh, R., et al. 2009. Autophagy regulates lipid metabolism. Nature. 458:1131-1135.

36. Piva, R., et al. 2006. Ablation of oncogenic ALK is a viable therapeutic approach for anaplastic largecell lymphomas. Blood. 107:689-697.

37. Schattenberg, J.M., et al. 2006. JNK1 but not JNK2 promotes the development of steatohepatitis in mice. Hepatology. 43:163-172.

38. Liu, H., et al. 2000. Inhibition of c-Myc expression sensitizes hepatocytes to tumor necrosis factor-induced apoptosis and necrosis. J. Biol. Chem. 275:40155-40162.
39. Komatsu, M., et al. 2005. Impairment of starvationinduced and constitutive autophagy in Atg7-deficient mice. J. Cell Biol. 169:425-434.

40. He, W., et al. 2003. Adipose-specific peroxisome proliferator-activated receptor $\gamma$ knockout causes insulin resistance in fat and liver but not in muscle. Proc. Natl. Acad. Sci. U. S. A. 100:15712-15717.

41. D’Ambra, R., Surana, M., Efrat, S., Starr, R.G., and Fleischer, N. 1990. Regulation of insulin secretion from beta-cell lines derived from transgenic mice insulinomas resembles that of normal beta-cells. Endocrinology. 126:2815-2822.
42. Matthews, D.R., et al. 1985. Homeostasis model assessment: insulin resistance and beta-cell function from fasting plasma glucose and insulin concentrations in man. Diabetologia. 28:412-419.

43. Wang, Y., Schattenberg, J.M., Rigoli, R.M., Storz, P., and Czaja, M.J. 2004. Hepatocyte resistance to oxidative stress is dependent on protein kinase Cmediated down-regulation of c-Jun/AP-1. J. Biol. Chem. 279:31089-31097.

44. Dohm, G.L., Huston, R.L., Askew, E.W., and Weiser, P.C. 1972. Effects of exercise on activity of heart and muscle mitochondria. Am. J. Physiol. 223:783-787. 